University of New Hampshire

University of New Hampshire Scholars' Repository

Center for Coastal and Ocean Mapping

Center for Coastal and Ocean Mapping

2006

\title{
Optimal Image Blending for Underwater Mosaics
}

\author{
Fan Gu \\ University of New Hampshire, Durham \\ Yuri Rzhanov \\ University of New Hampshire, Durham, Yuri.Rzhanov@unh.edu
}

Follow this and additional works at: https://scholars.unh.edu/ccom

Part of the Oceanography and Atmospheric Sciences and Meteorology Commons

\section{Recommended Citation}

Gu, Fan and Rzhanov, Yuri, "Optimal Image Blending for Underwater Mosaics" (2006). Center for Coastal and Ocean Mapping. 1159.

https://scholars.unh.edu/ccom/1159

This Poster is brought to you for free and open access by the Center for Coastal and Ocean Mapping at University of New Hampshire Scholars' Repository. It has been accepted for inclusion in Center for Coastal and Ocean Mapping by an authorized administrator of University of New Hampshire Scholars' Repository. For more information, please contact Scholarly.Communication@unh.edu. 


\title{
Optimal Image Blending for Underwater Mosaics
}

\author{
Fan Gu, YuriRzhanov
}

Center for Coastal and Ocean Mapping, University of New Hampshire, USA

\section{Email: \{fan, yuri\}@ccom.unh.edu}

\section{Introduction}

in the recent years, mosaics created from individual images acquired underwater are divided into three categories:

a. Targeting extraction of quantitative information (distances, sizes, shapes, etc.) local distortions.

c. Aiming at accurate recovery of three-dimensional information about the seafloor, is capable of achieving both goals, but requires principally different approach, and has substantially higher level of complexity.

uzzy and difficult to process. Problems can be classified in to three aspects:

a. Limited visibility underwater

b. Artificial spatially inhomogeneous illumination

c. Parallax issues

In this poster, we are not concerned with the ability to measure distances and sizes as accurately as possible. Algorithms for object recognition and shape extraction are typically and rapid changes in illumination. Our goal is:

present in the case of artificial lighting.

b. Combine individual image frames into a single mosaic in some optimal way. Note: "Optimal" may have different meanings depending on intended consumer: scientist, trying
to deduce large-scale interrelationships; computer program, extracting shapes according to some specific rule; or a high-school student learning about a deep-sea environment.

\section{Our Method}

The method proposed here based on the graph-cut in the gradient domain is to overcome the defects of the single graph-cut technique, which would have apparent seam when two images have inhomogeneous illumination, and the single gradient domain stitching, which can still
cause blurring in a misaligned case. In addition, it reduces the artifacts when combining these two methods directly (Fig. 10).

The procedure is as follows, assuming that two images have aiready been aligned and we take only one color channel for illustration (Fig.5):

1) Calculate the gradient values of two images

2) According to the overlapping area (which in general is an irregular polygon), a boundary box is obtained (fig. 6), which is composed of three parts. overlapping are, and pans that have
contributions from only one of the images. 3) Within the boundary box execute

(Fig. 7), using weighting function to smooth the boundary cut and and get the graph-cut mask domain mask (Fig. 8 ).

4) Fill in the boundary box with gradient values according to the mask matrix, and use it as a source term of the Poisson equation. Boundary values of the boundary box are from the ariginal pixel values of two inages given the boundary of the mast

volven equation with 6) Put the ry condition

(Fig

Transformations relating consecutive images are either deduced from the navigation tata estimated from the imagery. Frames are added sequentially to already existing mosaics.

\section{Previous Methods}

Current blending techniques can be divided in two main categories, assuming hat the images have already been aligned:

Optimal seam algorithm: between two overlapping images are minimal, each image is copied to the corresponding side of the seam. One simple and commonly used method is the winen the whe two inages are involved. As opposed to this "memoryless" approach, the images are needed to be mosaiced. However the seam may still be visible where brightness of neighboring original images differs dramatically.

By manipulating the images in transition region, two images are blended to release the illumination difference. Most common blending techniques employ simple averaging of images in the overlapping regions (Fig. 2). This results in ghosting antacts, blunkg, and visible seams that degrade the mosaic. Some Fig 3) which enploys the specia wophting functions multi- or alpha blending

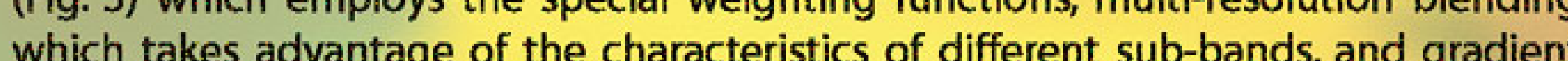
domain stitching (Fig 4) which is designed to remove sharp changes of brightness across the frame boundaries. However, blurring and ghosting effects could not be avoided due to misalignment of the underwater imagery.
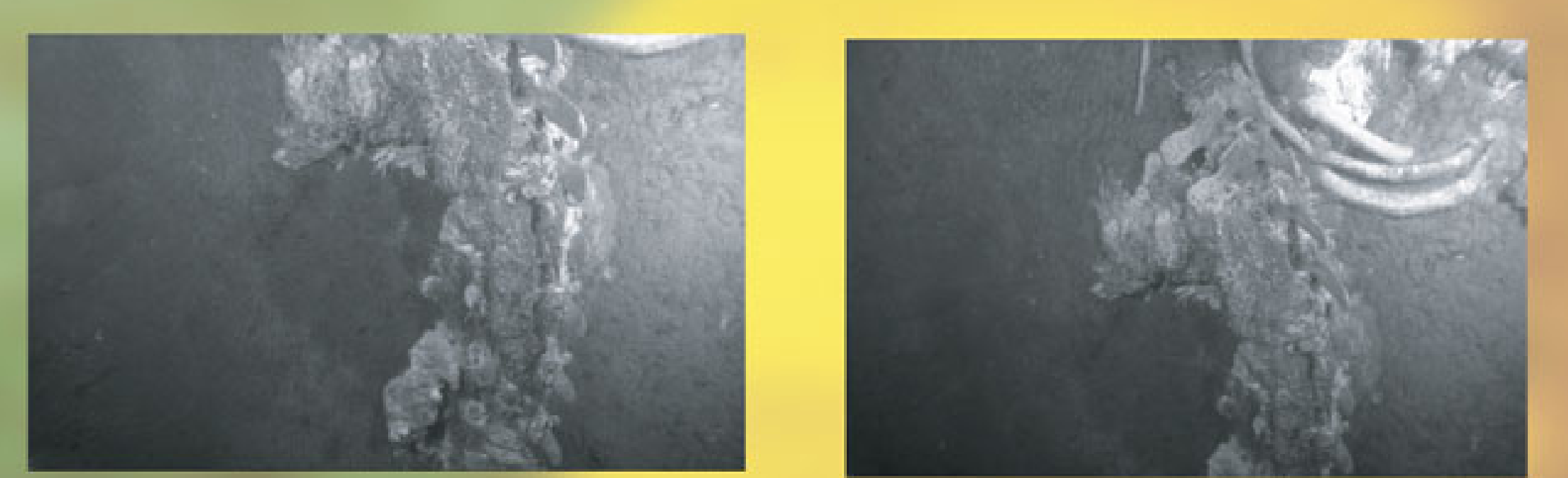

Fig. 5
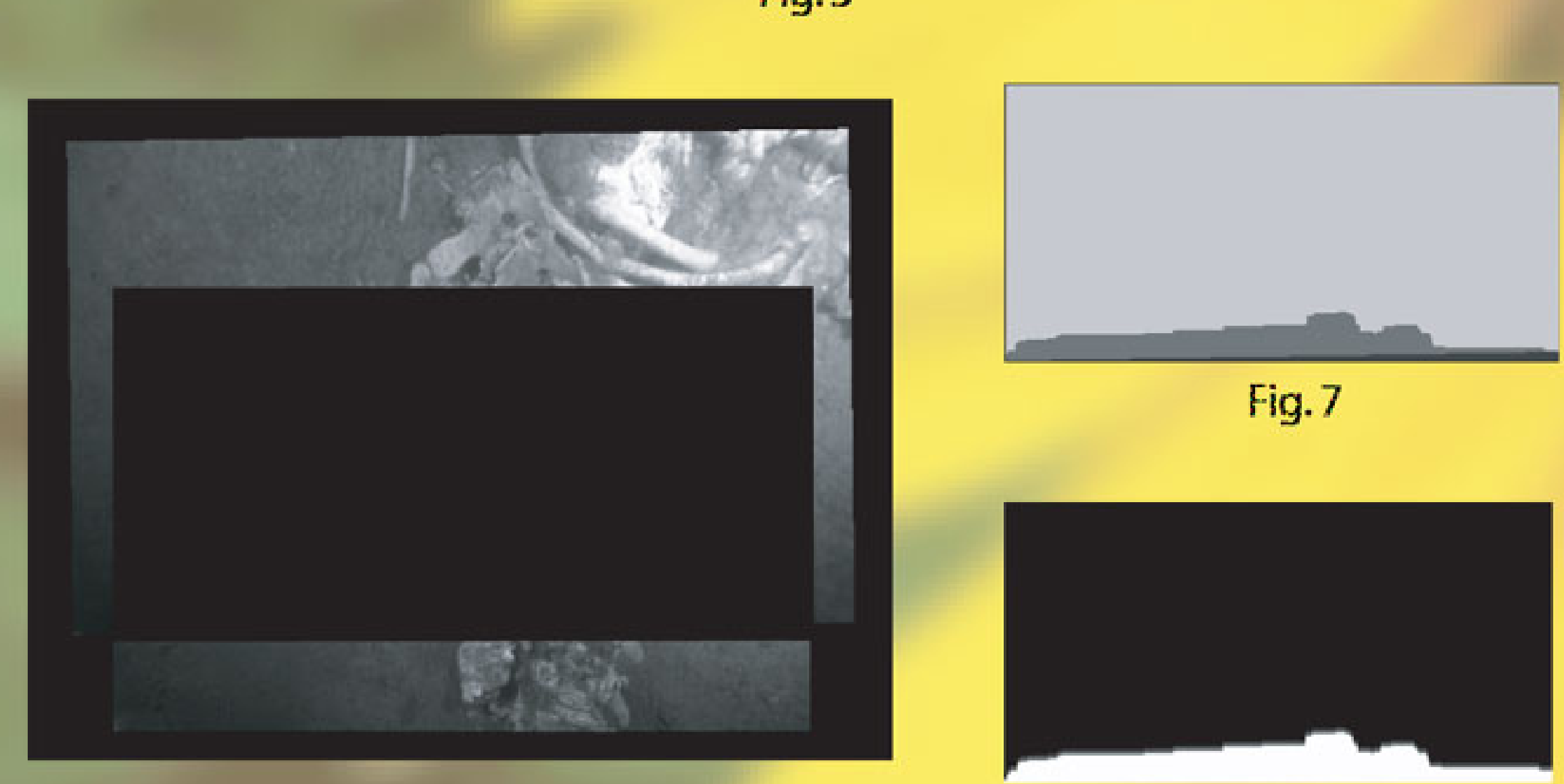

Fig. 7

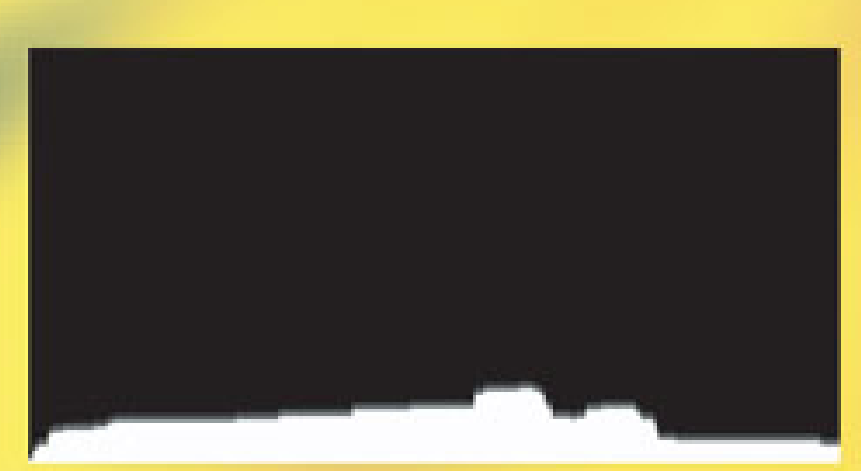

Fig. 8 b. Transition smoothing algorithm:

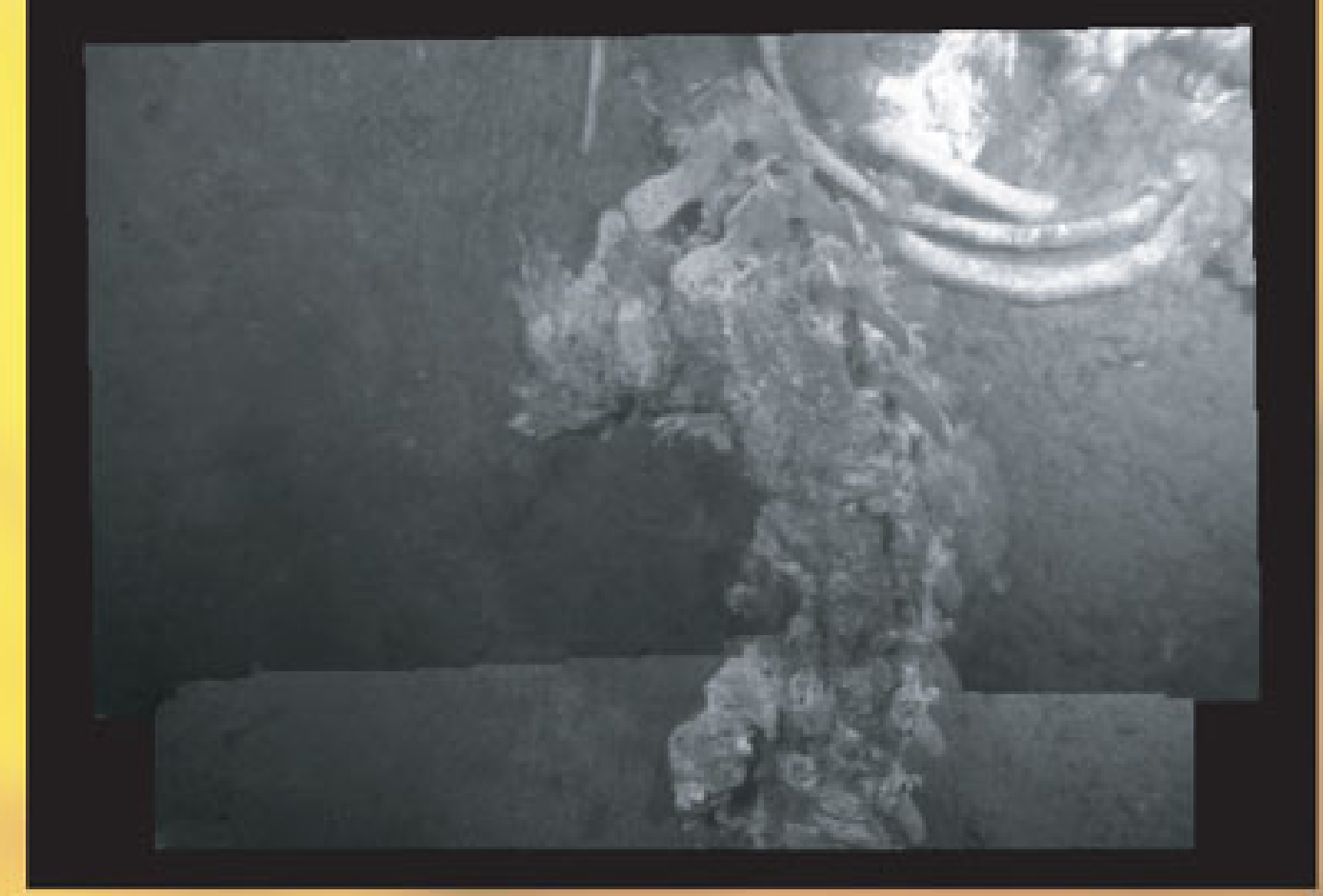

Fig. 1

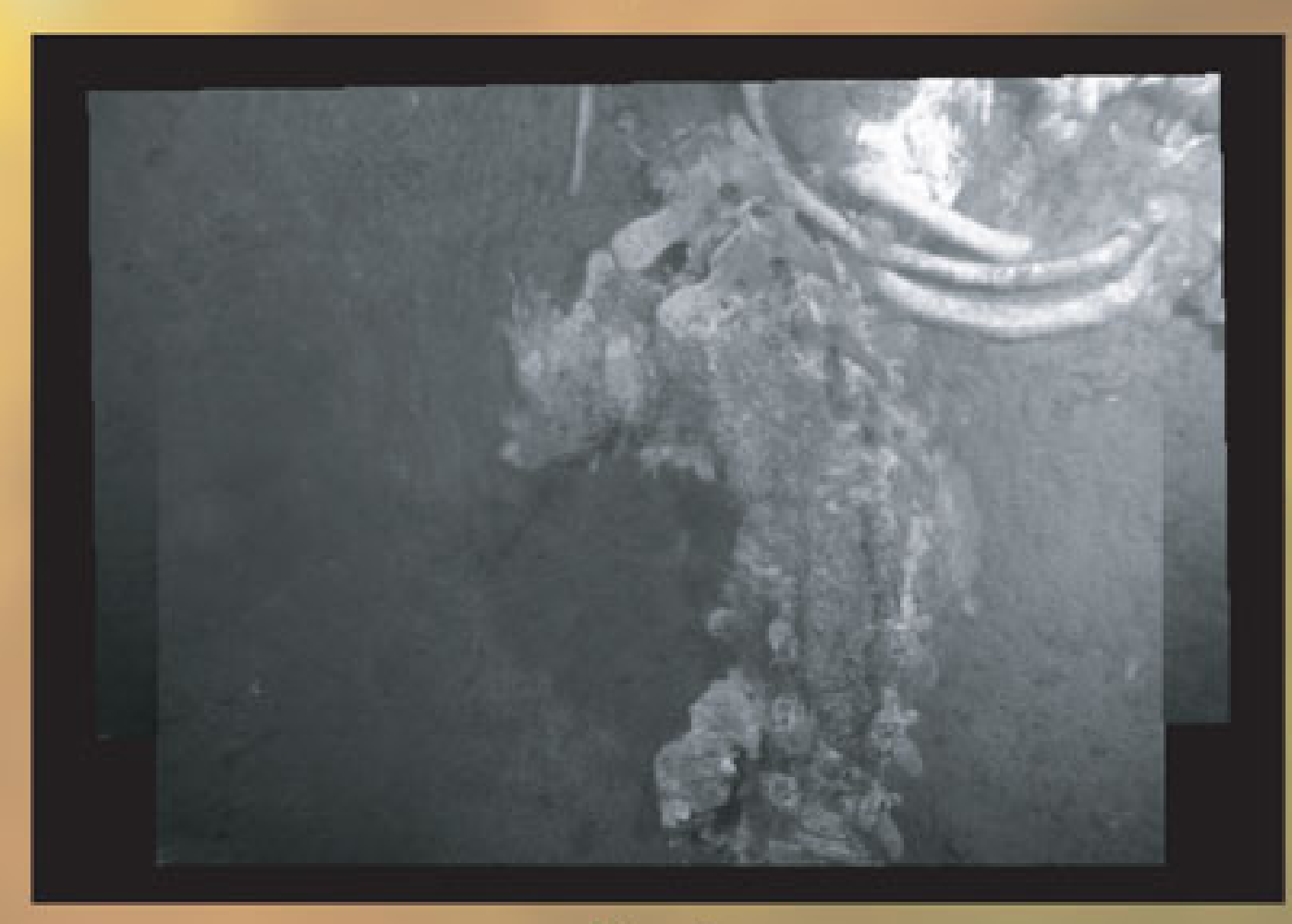

Fig. 3

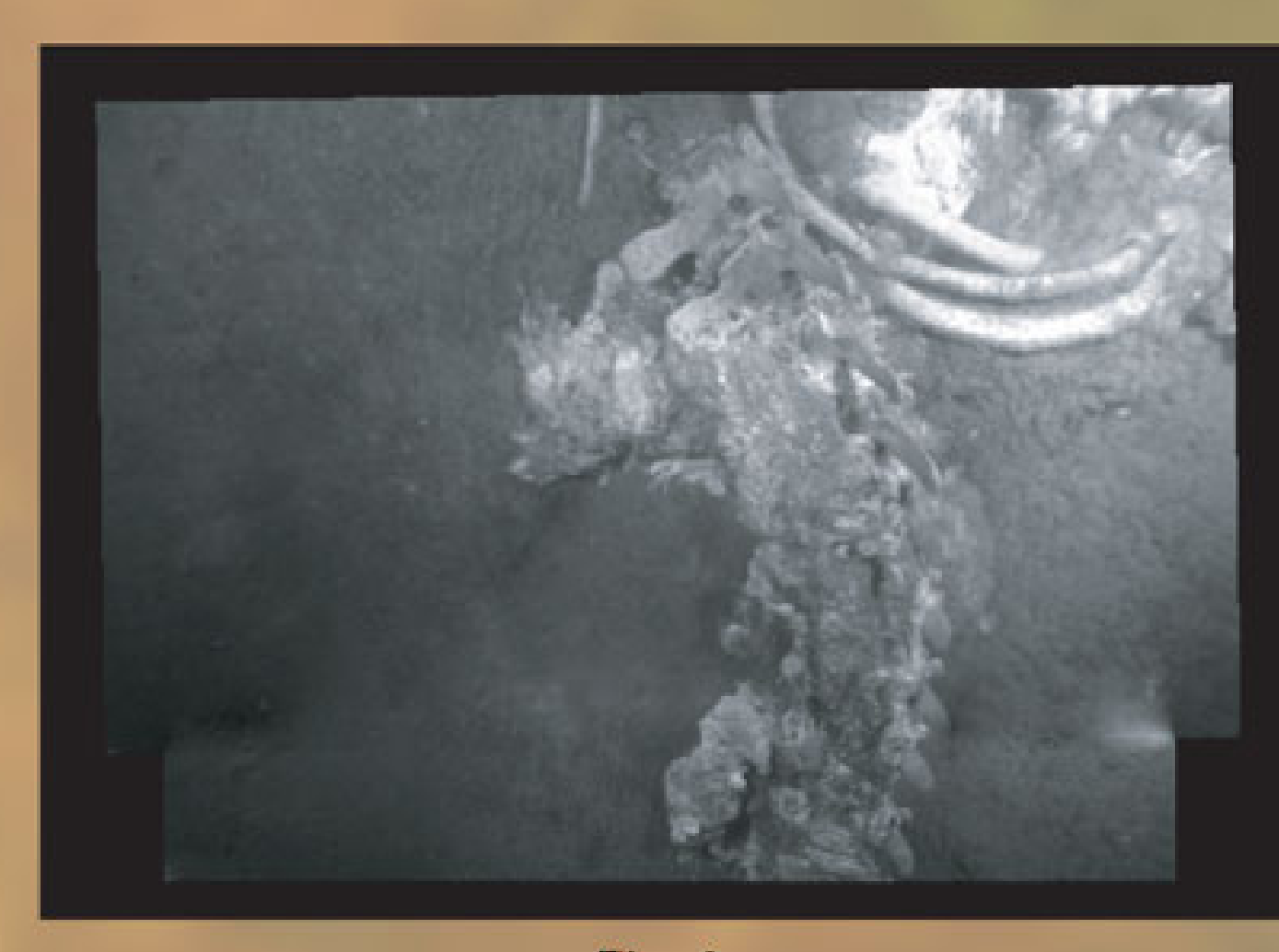

Fig. 9

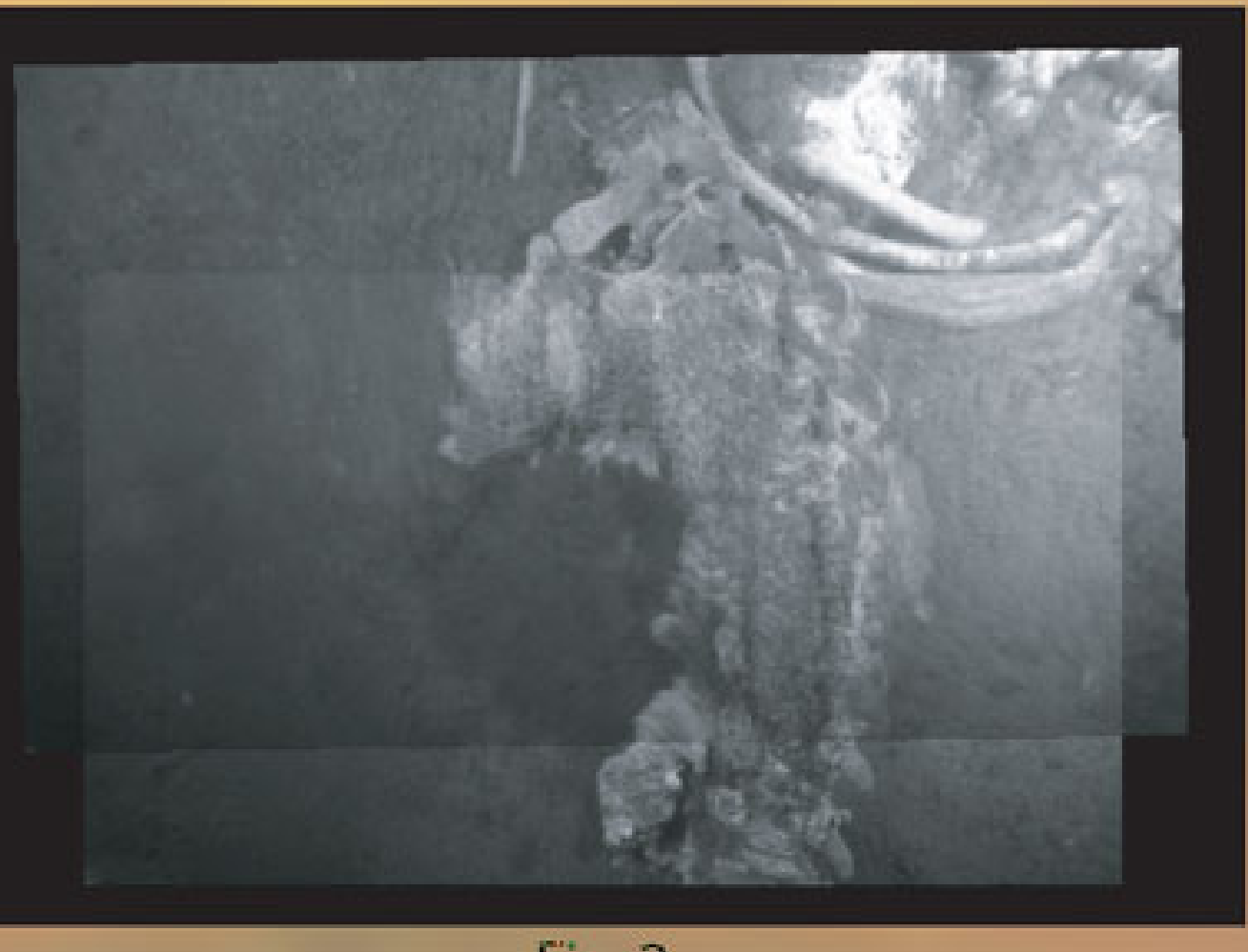

Fis 2
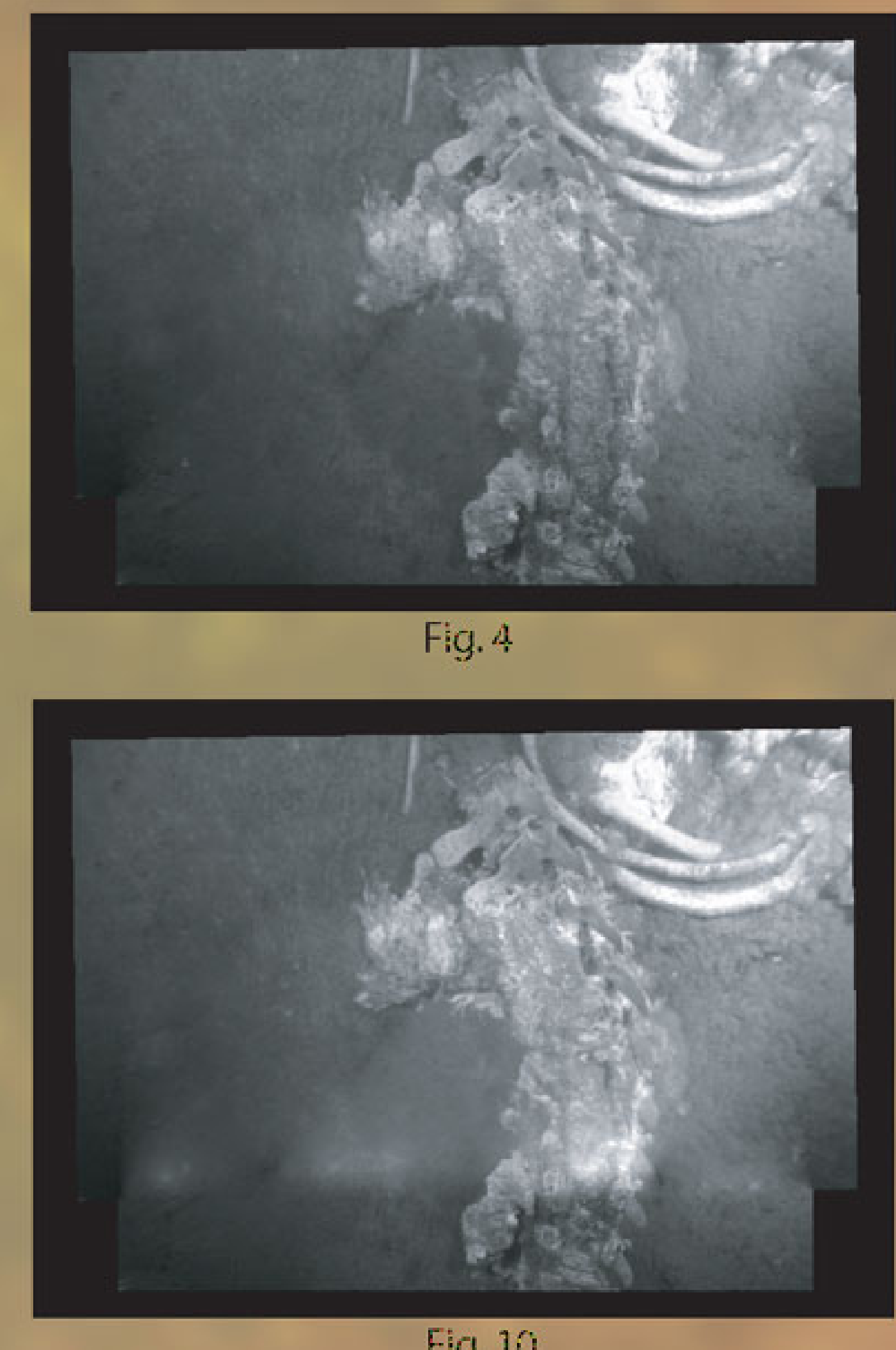

Fig. 10

Comclution

Due to the artificial lighting and 30 content of imaged terrain, imagery taken underwater almost always suffers from inhomogeneous mumination and feature misalignment, when mosaced. This causes degradation of the final product and makes it more difficult to post-process. Often used mean value averaging blending technique an hardly salisly dhe demand of post processing such as feature image processing. Most of them fail when it comes to the underwater images, which have different specifics. We reviewed the existing popular methods and combined them in a way to facilitate in postprocessing of underwater mosaics. Experimental results show the effectiveness of the proposed methods, comparing with other existing methods. The reason for artifacts, occasionally occurring in the reconstructed process, requires further investigation. 